\title{
Anti-DLL4/VEGF Bispecific Monoclonal Antibody OMP-305B83
}

National Cancer Institute

\section{Source}

National Cancer Institute. Anti-D LL4IVEGF Bispecific Monoclonal Antibody OMP-305B83.

NCI Thesaurus. Code C119620.

A bispecific monoclonal antibody directed against both the Notch ligand delta-like 4 (DLL4) and the human tyrosine kinase vascular endothelial growth factor (VEGF), with potential anti-angiog enic and antineoplastic activities. The anti-DLL4 moiety of antiDLL4/VEGF monoclonal antibody OMP-305B83 specifically binds to DLL4, prevents its interaction with Notch receptors, and inhibits Notch-mediated signaling and gene transcription, which may both block tumor angiogenesis and inhibit tumor cell growth. The anti-VEGF moiety binds to VEGF and prevents the binding of VEGF to its receptor, which blocks VEGF-mediated signaling and further inhibits the growth and maintenance of tumor blood vessels. The expression of DLL4 is highly restricted to the vascular endothelium; DLL4/Notch signaling is required for the development of functional tumor blood vessels. The expression of the pro-angiog enic growth factor VEGF is associated with tumor angiogenesis and tumor cell proliferation and invasion. 